MATEC Web of Conferences 13, 02018 (2014)

DOI: $10.1051 /$ matecconf/ 20141302018

(C) Owned by the authors, published by EDP Sciences, 2014

\title{
Natural Convection-Radiation from a Vertical Base-Fin Array with Emissivity Determination
}

\author{
Viswanatha Sharma Korada a , Mark Ovinis, Suhaimi B Hassan \\ Department of Mechanical Engineering, Universiti Teknologi PETRONAS, Seri Iskandar, \\ 31750 Tronoh, Perak, Malaysia
}

\begin{abstract}
Experiments have been conducted to determine the emissivity for black chrome coated and uncoated aluminum surfaces. The emissivity of the surfaces is estimated considering combined convection radiation heat transfer and observed to be a constant in the range of 60 to $110^{\circ} \mathrm{C}$. The combined heat transfer coefficients from black chrome coated vertical base vertical fin array of size $70 \times 70 \mathrm{~mm}$ consisting of 22 aluminum fins with a fin spacing of $10 \mathrm{~mm}$ by natural convection and radiation has been determined at different heat inputs. Theoretical analysis of single fin geometry of constant thickness considering both convection and radiation has been used to predict the temperature distribution and heat flow. The theoretical values of heat flow estimated for a fin array is in good agreement with the experimental observations validating the emissivity of the surface. The experimental data is further validated with the equations of Nusselt presented by Churchill and Chu.
\end{abstract}

\section{Introduction}

Miniaturization of integrated circuits and reduction of spacing between chips has contributed to significant improvement in the performance of computer systems to meet high power dissipation requirement. The temperature of these components is controlled by forcing air over the surface. However, surfaces are cooled by natural convection for trouble-free and noiseless operation, as in the case of cooling of certain electronic equipment, in room heating or special heat exchange process. Coated finned surfaces are commonly employed for enhancement of heat dissipation by combined convection and radiation. The contribution of radiation from the hot surface to the ambient may account for more than $20 \%$ of the total heat dissipated.

Early experiments to estimate heat dissipation by free convection from parallel plate geometry has been undertaken by Elenbaas [1]. Laminar natural convection from a vertical plate subjected to uniform surface heat flux has been presented by Sparrow and Gregg [2] using similarity technique. The effect of staggering of fins has been studied by Sobel et al. [3]. The condition for optimum spacing to minimize temperature difference between the plate and the fluid has been presented by Levy [4]. Using the Elenbaas correlation, Bar-cohen [5] analysed an array of longitudinal fins to determine optimal spacing and thickness for maximum heat dissipation. Bar-cohen and Jelinek [6] showed that the fin spacing should equal the fin thickness for optimum material of the fin. Numerical

This is an Open Access article distributed under the terms of the Creative Commons Attribution License 2.0, which permits unrestricted use, distribution, and reproduction in any medium, provided the original work is properly cited. 
analyses with natural convection between vertical parallel plate configurations have been made by Leung et al. [7]. Sunil and Sobhan [8] considered the effect of variable thermal conductivity on the average heat transfer coefficient for a given fin height. An increase in the base temperature of the fin resulted in an increase in the average heat transfer coefficient, the range of which is influenced by the fin material. Dayan et al. [9] studied the contribution of thermal radiation in the total fin array cooling capacity. They concluded that the inclusion of radiation influence optimal spacing between fins which is pronounced when the surface emissivity is low. When radiation becomes strong, owing to large surface emissivity, the fin spacing is weakly affected by the radiation component. They observed that the surface temperature has no significant influence on the optimal fin spacing and attributed to the fact that at higher temperature, buoyancy can still drive an effective convective flow through tighter channels. A long channel presents considerable flow resistance and therefore should be compensated by wider spacing between fins. A significant outcome of the investigation is that the optimal fin spacing lies in a very narrow range for a wide variety of array geometries, the channel length is identified as the most significant parameter. Studies are undertaken to determine the optimum spacing between fins, thickness of the fins for minimum mass, the effect of fin thermal conductivity, temperature distribution in fin, combined heat transfer coefficient on heat dissipation capability of a fin array system. In most of the analyses, the contribution of radiation is estimated indirectly as the difference between the combined heat loss and convection loss estimated with equations. The emissivity of the surface is required to validate the heat loss by radiation. Hence, an experimental setup is fabricated to determine the emissivity of chrome coated surface commonly used in fin arrays for cooling application.

An equation considering convection and radiation for a fin of uniform cross section is deduced from the energy balance relation. The equation is solved subject to boundary conditions to obtain the temperature variation, local and average heat transfer coefficients for various operating conditions. The influence of emissivity, convection heat transfer, and ambient temperature on overall heat transfer coefficients is determined and compared with the experimental data undertaken with a fin array setup.

\section{Experimental determination of emissivity}

To determine the emissivity at different surface temperatures, an experimental setup consisting of a strip heater sandwiched between two aluminum plates of dimensions $100 \times 200 \times 4 \mathrm{~mm}$ is fabricated. The assembly is located in a wooden box with aluminum plates held in vertical orientation. The plate inside the box is supported with epoxy resin plate of $5 \mathrm{~mm}$ thick and rock wool is packed between the resin plate and the wooden box to obtain negligible heat loss from the rear. Feasibility to replace the uncoated surface with a black chrome coated surface is made. This enabled determination of the surface temperatures with the aid of thermocouples for both conditions. A control panel consisting of voltmeter, ammeter, temperature indicator, dimmerstat and thermocouple selector switch for obtaining pertinent information is provided for estimating the emissivity of the surfaces. The input power to the heater is varied to obtain plate temperature at steady state. The thermocouples located at the four corners and at the centre of the surface showed identical temperatures. The values are validated with the temperatures measured with IR thermometer with a deviation of $\pm 1.0^{\circ} \mathrm{C}$. Average Nusselt numbers are calculated using theoretical equations for the vertical isothermal flat plate available in literature for both the uncoated and black chrome coated surfaces. Using these values, the heat leaving the surface by convection and hence by radiation is evaluated. It is estimated from the calculations of heat loss by radiation, the emissivity of the coated surface to be 0.7 in the temperature range of $60-$ 
$110^{\circ} \mathrm{C}$. Hence this value is taken for comparison of the heat flow by radiation in the fin array experiments.

\section{Experiments with fin array setup}

An enclosure to dissipate heat by natural convection generated by a pulsating electronic component is simulated by providing a heating element inside the enclosure with integral fins on one side as shown in Fig. 1 and closed with a cover on the other. Such enclosure/casing is generally used for locating electronic gadgets for operation in remote places and designed for cooling by passive means. The size of the enclosure for locating the components as per the design requirement is $530 \mathrm{x} 360 \mathrm{x} 140 \mathrm{~mm}$ with a threshold temperature of $120^{\circ} \mathrm{C}$ to be ensured for smooth functioning of the electronic components. The enclosure is designed to have 6 rows, each row consisting of 22 vertical square fins of $5 \mathrm{~mm}$ thick $(\mathrm{t}), 70 \mathrm{~mm}$ length $(\mathrm{H})$ and protruding $70 \mathrm{~mm}$ from vertical base (L) with a fin spacing (s) of $10 \mathrm{~mm}$ and black coated. A gap width of $25 \mathrm{~mm}$ is provided between two successive rows. Three strip heaters connected in series each of $400 \mathrm{~W}$ maximum rating is located inside the enclosure at the base of the fins to simulate heat generation by electronic components as shown in Fig.1. The rectangular enclosure is made of aluminum for ease of fabrication with the base unit being cast. The cast aluminum block is machined for obtaining the fin array. The enclosure is put into simulated operating conditions with the base and fin array in vertical orientation. The amount of heat supplied at the base is altered by varying the current to the heaters using a voltage regulator at the control panel. The input power is measured using calibrated digital ammeter and voltmeter at steady state. Precalibrated copper constantan thermocouples with an accuracy of $0.1{ }^{\circ} \mathrm{C}$ are fixed to the enclosure, fin base and fin tip at different rows and connected to a digital temperature indicator. The temperature from these locations is recorded at different heat inputs. The relatively high thermal conductivity of aluminum facilitated the achievement of almost uniform temperature at the air-base interface of the fin array and the enclosure surface exposed to atmosphere. Experiments are undertaken with black chrome coated fin array system.

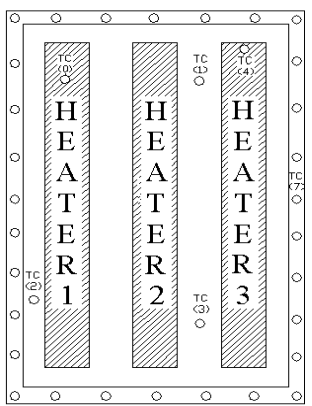

CROSS SECTTONAL FRONT YIEW

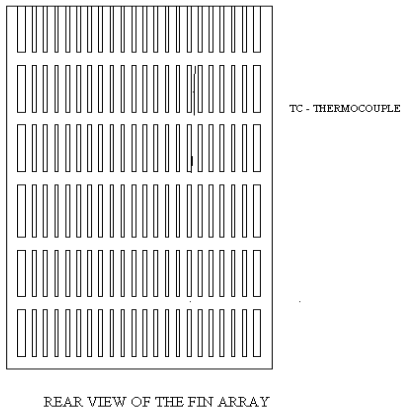

REAR VIEW OF THE FIN ARRAY

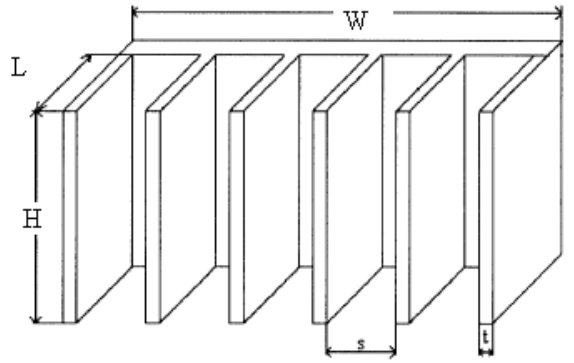

Figure 2. Fin Geometry

Figure 1. Schematic diagram of the experimental setup

\subsection{Analysis of fin considering radiation}

One-dimensional steady heat conduction is assumed to be valid for the configuration of the fin shown in Fig.2. To determine the differential equation that will yield the fin temperature as a function of $x$ along L, an energy balance is made on a differential element of width $d x$, of uniform cross section area, $A=H t$.

$$
\frac{d}{d x}\left(t \frac{d T}{d x}\right) d x=\frac{2}{k}\left\{h\left(T-T_{\infty}\right)+\varepsilon \sigma\left(T^{4}-T_{\infty}^{4}\right)\right\}
$$


The governing differential equation in non-dimensional form is obtained as

$$
\frac{d^{2} \theta}{d \xi^{2}}-N_{R}\left[N_{c} \theta+\left\{(\theta+\psi)^{4}-\psi^{4}\right\}\right]=0
$$

subject to boundary conditions:

$$
\begin{aligned}
& \text { At } \xi=0, \quad \theta=1 ; \xi=1, \quad \frac{d \theta}{d \xi}=0 \\
& Q=\left.k_{a l} A \frac{d \theta}{d \xi}\right|_{\xi=0}
\end{aligned}
$$

An iterative procedure is undertaken for various input values of $R a_{L}, N_{C}, N_{R}$ in the experimental range for different values of temperature ratio term $\psi$ and emissivity $\varepsilon$ of the test surface. The temperature distribution and the local heat transfer coefficient are evaluated using Eq. (2a) subject to the boundary conditions (2b). The average heat transfer evaluated from numerical results for a fin is used to estimate the heat flow from the fins. The equations presented by Yuncu and Kakac [11] are used for comparison.

Churchill and Chu's first relation [11]

$N u_{H}=\left[0.825+\left(0.387 R a_{H}^{1 / 6}\right) /\left\{1+(0.492 / \mathrm{Pr})^{1 / 6}\right\}^{8 / 27}\right]^{2} \quad$ for $10^{-1}<R a_{H}<10^{12}$

Churchill and Chu's second relation [11]

$$
N u_{H}=\left\{0.68+0.670\left(R a_{H}\right)^{1 / 4}\right\} /\left\{1+(0.492 / \mathrm{Pr})^{1 / 6}\right\}^{4 / 9} \quad \text { for } 10^{-1}<R a_{H}<10^{9}
$$

McAdam's relation [11]

$$
N u_{H}=0.59\left(R a_{H}\right)^{1 / 4} \quad \text { for } 10^{4}<R a_{H}<10^{9}
$$

Churchill and Usagi's relation [11]

$$
N u_{H}=0.670\left(R a_{H}\right)^{1 / 4} /\left\{1+(0.492 / \mathrm{Pr})^{9 / 16}\right\}^{4 / 9} \quad \text { for } 10^{5}<R a_{H}<10^{9}
$$

\section{Results and discussion}

The temperature distribution in the fin and variation of local heat transfer coefficient for different values of radiation parameter $N_{R}$ and temperature ratio term $\psi$ is shown in Figs 3 and 4 . Evidently with increase in $N_{R}$, heat dissipation is higher and consequently lower wall temperatures and higher values of heat transfer coefficients can be expected. In Fig.4 the influence of emissivity on local heat transfer coefficient is shown. The influence of $\varepsilon$ is pronounced at higher values of $\psi$. An increase in the value of $\psi$ implies lower values of temperature difference between the wall and the ambient and consequent higher values of heat transfer coefficient for a given input $Q$. The influence of convection parameter, $N_{C}$ on the average heat transfer coefficient can be observed from Fig.5. Increase in the value of $N_{C}$ implies greater heat loss by convection which is evident from the graph drawn between average heat transfer coefficients and $N_{R}$ as shown in Fig.5. The estimated value of heat flow from the fin array at different operating temperatures is shown in Fig. 6 along with the experimental data. It can be observed that the estimated values of heat flow increases with temperature which is in good 
agreement with the theoretical values drawn with emissivity, $\varepsilon=0.7$. To further test the validity of the present analysis, the experimental values of heat dissipated $Q_{\exp }$ are compared with values from theory as shown in Fig.7. Equations (3) to (6) and the data of Guvenc and Yuncu [10] presented by Yuncu and Kakac [11] is in good agreement compared with the numerical results as shown in Fig.8, thus confirming the applicability of single fin analysis with radiation in the design of fin array system.

\section{Conclusions}

a) The theoretical analysis of single fin is observed to predict the heat loss from 22 fins having $10 \mathrm{~mm}$ spacing with 6 rows in the experimental range of $38<Q<99 ; \quad 3.93<\psi<8.17$; $334<T_{b}<378$;

b) The ratio of ambient temperature to the excess temperature, (difference between base and ambient) represented by the temperature ratio term $\psi$ is observed to be a significant parameter along with radiation term $N_{R}$ in the evaluation of heat transfer coefficient.

c) A value of 0.7 for emissivity of the black chrome surface in the temperature range of $60-110^{\circ} \mathrm{C}$ is found to correlate well with other authors and the experimental data obtained.

d) The analysis of single fin with the inclusion of radiation can be used to estimate heat flow from a fin array.

\section{References}

1. Elenbaas, W., Physica, 9, 1 - 28. (1942)

2. Sparrow, E. M., and Gregg, J. L., Transactions of ASME, 78, 435 - 440. (1956)

3. Sobel, N., Landis, F., and Muller, W.K., Proc. $2^{\text {nd }}$ Int. Heat Transfer Conference, 2, 121-125. (1966)

4. Levy, K., Transactions of ASME J. Heat Transfer, pp. 463 - 465. (1971)

5. Bar - Cohen, A., Trans. of ASME, 101, pp. 564 - 566. (1979)

6. A.Bar-Cohen, M.Jelinek, 150-120, Hemisphere, New York. (1990)

7. Leung, C.W., Probert S.D and Rapely C.W., Applied Energy, 253 - 265. (1990)

8. Sunil and Sobhan C.B., Int. Journal of Heat Fluid Flow, 14, 191-200 (1993).

9. Dayan.A, Kushnir.R., Mittelman, G., and Ullmann, A., 47, pp.2849 - 2860. (2004)

10. A.Guvenc, H.Yuncu, J. Heat and Mass Transfer, 37, 409-416. (2001)

11. Yuncu H, S Kakac, Basic Heat Transfer (in Turkish) Bilim Yayincilik Ltd., Sti, Ankara. (1999)

\section{Nomenclature}

$N_{c} \quad$ Convection parameter, $h / \varepsilon \sigma\left(T_{b}-T_{\infty}\right)^{3}$

$N u_{H}$ Nusselt number, $h . H / k$

$Q$ heat flow, $\mathrm{W}$

$\psi \quad$ temperature ratio term, $T_{\infty} /\left(T_{b}-T_{\infty}\right)$

$\xi \quad$ dimensionless distance, $x / L$
$N_{R}$ Radiation parameter, $2 \varepsilon \sigma L^{2}\left(T_{b}-T_{\infty}\right)^{3} /\left(k_{a l} t\right)$

$\operatorname{Pr} \quad$ Prandtl number of air, $v / \alpha$

$R a_{H} \quad$ Rayleigh number, $\left\lfloor g \beta\left(T_{b}-T_{\infty}\right) H^{3} / v^{2}\right\rfloor \operatorname{Pr}$

$\theta$ dimensionless temperature, $\left(T-T_{\infty}\right) /\left(T_{b}-T_{\infty}\right)$

$\infty$ ambient 


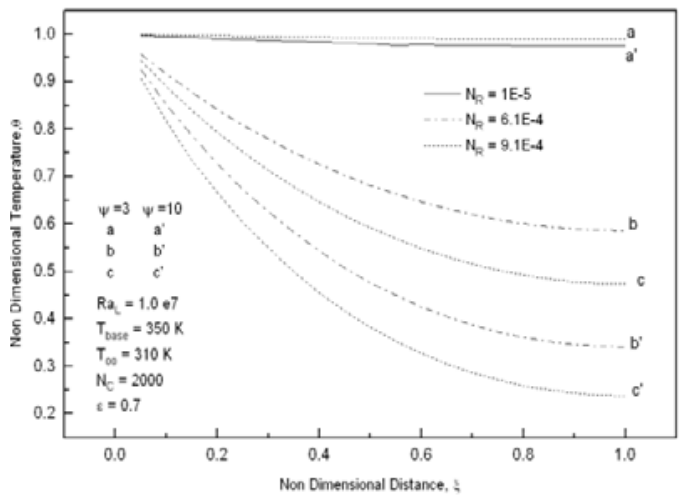

Figure 3. Local temperature variation for different values of $N_{R}$ and $\psi$

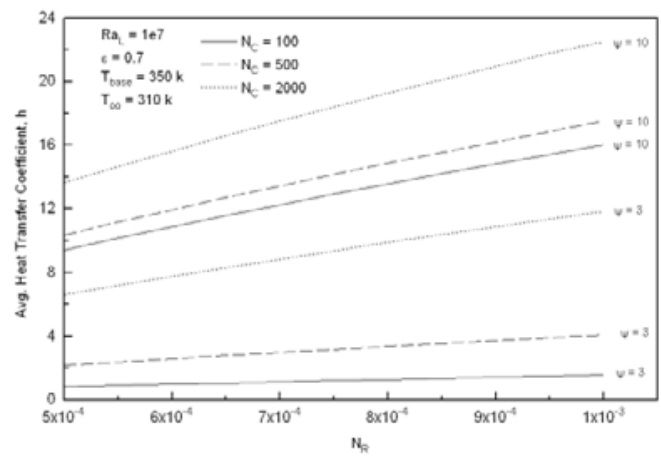

Figure 5. Variation of average heat transfer Coefficient with $N_{R}$ for different values of $N_{c}$

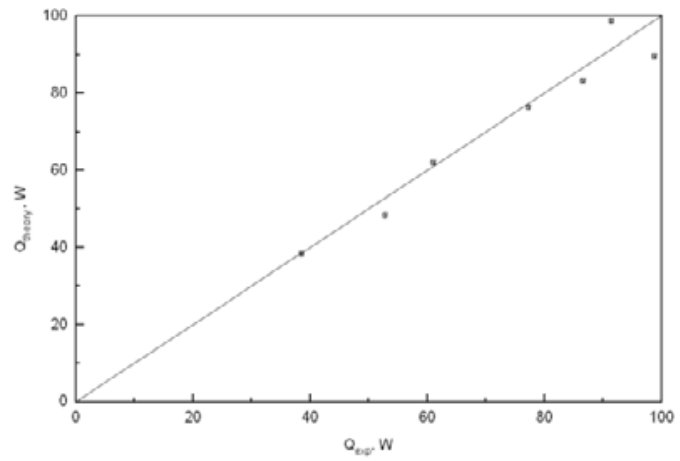

Figure 7. Comparison of experimental data with values estimated from theory

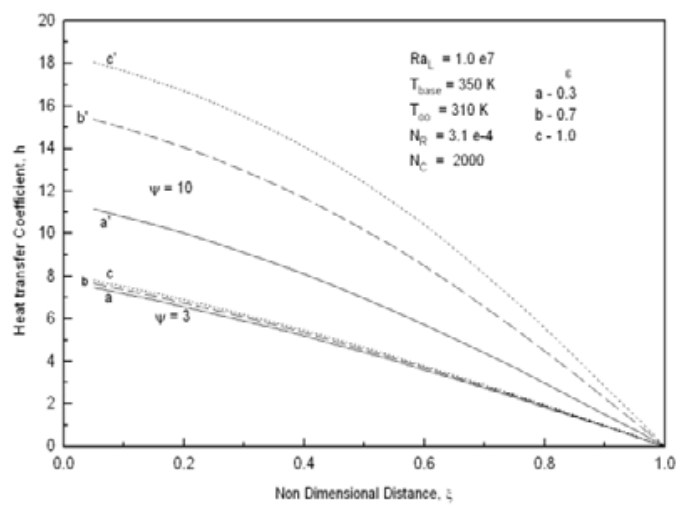

Figure 4. Variation of local overall heat transfer coefficient for different emissivities

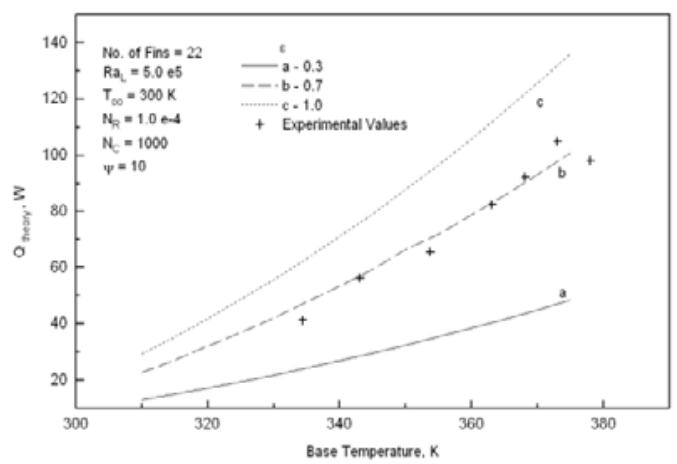

Figure 6. Predicted values of heat loss from theory at various temperatures

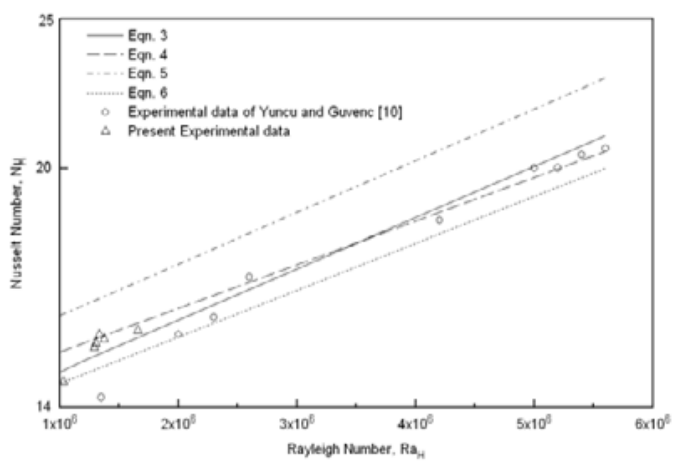

Figure 8. Comparison of experimental Nusselt with correlations from the literature 